\title{
The Second Variation of the Functional $L$ of Symplectic Critical Surfaces in Kähler Surfaces
}

\author{
Xiaoli Han · Jiayu Li
}

Received: 5 November 2014 / Accepted: 23 December 2014 / Published online: 19 February 2015

(C) School of Mathematical Sciences, University of Science and Technology of China and Springer-Verlag Berlin Heidelberg 2015

\begin{abstract}
Let $M$ be a complete Kähler surface and $\Sigma$ be a symplectic surface which is smoothly immersed in $M$. Let $\alpha$ be the Kähler angle of $\Sigma$ in $M$. In the previous paper Han and Li (JEMS 12:505-527, 2010) 2010, we study the symplectic critical surfaces, which are critical points of the functional $L=\int_{\Sigma} \frac{1}{\cos \alpha} d \mu$ in the class of symplectic surfaces. In this paper, we calculate the second variation of the functional $L$ and derive some consequences. In particular, we show that, if the scalar curvature of $M$ is positive, $\Sigma$ is a stable symplectic critical surface with $\cos \alpha \geq \delta>0$, whose normal bundle admits a holomorphic section $X \in L^{2}(\Sigma)$, then $\Sigma$ is holomorphic. We construct symplectic critical surfaces in $\mathbf{C}^{2}$. We also prove a Liouville theorem for symplectic critical surfaces in $\mathbf{C}^{2}$.
\end{abstract}

Keywords Symplectic critical surface $\cdot$ Holomorphic curve $\cdot$ Kähler surface

Mathematics Subject Classification $53 \mathrm{C} 42 \cdot 58 \mathrm{~J} 35$

\footnotetext{
X. Han $(\varangle)$

Department of Mathematical Sciences, Tsinghua University, Beijing 100084,

People's Republic of China

e-mail: xlhan@math.tsinghua.edu.cn

J. Li

School of Mathematical Sciences, University of Science and Technology of China, Hefei 230026, People's Republic of China

J. Li

Academy of Mathematics and Systems Sciences, Chinese Academy of Sciences, Beijing 100080, People's Republic of China

e-mail: lijia@amss.ac.cn
} 


\section{Introduction}

Let $M$ be a complete Kähler surface and $\Sigma$ be a surface immersed in $M$. One defines a metric on $\Sigma$ induced from $M$. The Kähler angle [2] $\alpha$ of $\Sigma$ in $M$ is defined by

$$
\left.\omega\right|_{\Sigma}=\cos \alpha d \mu_{\Sigma}
$$

where $d \mu_{\Sigma}$ is the area element of $\Sigma$ and $\omega$ is the Kähler form on $M$. We say that a surface $\Sigma$ is symplectic if $\cos \alpha>0$ on $\Sigma$. In [3], we introduce the functional

$$
L=\int_{\Sigma} \frac{1}{\cos \alpha} d \mu_{\Sigma}
$$

in the class of symplectic surfaces in $M$. It is clear that holomorphic curves minimize the functional. We call a critical point of the functional $L$ a symplectic critical surface. In [3], we calculate the first variation formula of the functional $L$ and derive the Euler-Lagrange equation, it is

$$
\cos ^{3} \alpha H-\left(J(J \nabla \cos \alpha)^{\top}\right)^{\perp}=0,
$$

where $H$ is the mean curvature vector of $\Sigma$ in $M$, and ()$^{\top}$ denotes tangential components of () and ()$^{\perp}$ denotes the normal components of (). We checked that it is an elliptic equation. We showed that [3], if $M$ is a Kähler-Einstein surface with nonnegative scalar curvature, each compact symplectic critical surface is holomorphic.

The main purpose of this paper is to compute the second variation formula of the functional $L$ of a symplectic critical surface. In particular, let $X$ be a normal variation with compact support, and let $Y=-J_{v} X$, then the second variation of the functional $L$ is

$$
\begin{aligned}
I I(X)+I I(Y)= & -4 \int_{\Sigma} \frac{R|X|^{2} \sin ^{2} \alpha}{\cos \alpha} d \mu_{\Sigma}+2 \int_{\Sigma} \frac{|\bar{\partial} X|^{2}\left(1+\cos ^{2} \alpha\right)}{\cos ^{3} \alpha} d \mu_{\Sigma} \\
& -2 \int_{\Sigma} \frac{\left(1+\cos ^{2} \alpha\right)}{\cos ^{5} \alpha}|X|^{2}|\nabla \alpha|^{2} d \mu_{\Sigma} .
\end{aligned}
$$

It follows from the formula clearly that if the scalar curvature of $M$ is positive, $\Sigma$ is a stable symplectic critical surface in $M$ with $\cos \alpha \geq \delta>0$, whose normal bundle admits a holomorphic section $X \in L^{2}(\Sigma)$, then $\Sigma$ is holomorphic. In the computation, we used techniques in [4].

In the last section, we study symplectic critical surfaces in $\mathbf{C}^{2}$. We construct symplectic critical surfaces in $\mathbf{C}^{2}$. The first example is a rotational symmetric surface $z=-\frac{1}{2} \log \left(x^{2}+y^{2}\right)$ in $\mathbf{R}^{3}$ which we see as a surface in $\mathbf{C}^{2}$. It is a simply connected complete symplectic critical surface with Gauss curvature close to -1 at one end and close to 0 at the other end, which is not holomorphic. We also prove a Liouville theorem for symplectic critical surfaces in $\mathbf{C}^{2}$. If $\Sigma$ is a complete symplectic critical surface in $\mathbf{C}^{2}$ with area quadratic growth, and $\cos \alpha \geq \delta>0$, then it is a holomorphic curve. 


\section{Preliminaries}

In this section, we recall the geometry of the normal bundle $v$ of $\Sigma$ in $M$ (see [4]) and fix our notation. We choose a local orthonormal frames $\left\{e_{1}, e_{2}, e_{3}, e_{4}\right\}$ in $M$ such that $\left\{e_{1}, e_{2}\right\}$ is a local orthonormal frames of $\Sigma$ and $\left\{e_{3}, e_{4}\right\}$ is a local orthonormal frames of the normal bundle $v$ of $\Sigma$ in $M$. We shall use the following conventions on the range of the indices,

$$
\begin{aligned}
& 1 \leq i, j, k \leq 2 \\
& 1 \leq A, B, C, D \leq 4 \\
& 3 \leq \lambda, \mu \leq 4 .
\end{aligned}
$$

Let $\left\{\theta^{A}\right\}$ be the dual frame of $\left\{e_{A}\right\}$, then the structure equations of $M$ are

$$
\begin{aligned}
d e_{A} & =\theta_{A B} \otimes e_{B}, \quad \theta_{A B}+\theta_{B A}=0, \\
d \theta_{A B} & =\theta_{A C} \wedge \theta_{C B}+\Phi_{A B}, \quad \Phi_{A B}=\frac{1}{2} R_{A B C D} \theta_{C} \wedge \theta_{D} .
\end{aligned}
$$

The induced connection one form $\theta_{i j}$ of $\Sigma$ is determined by

$$
\nabla e_{i}=\theta_{i j} \otimes e_{j} .
$$

The induced connection one form $\theta_{\lambda \mu}$ of $\nu$ is determined by

$$
\nabla e_{\lambda}=\theta_{\lambda \mu} \otimes e_{\mu} .
$$

Note that $\left(\theta_{i j}\right)$ defines a connection in the tangent bundle $T \Sigma$, hence a connection in the cotangent bundle $T^{\star} \Sigma$ and $\left(\theta_{\lambda \mu}\right)$ defines a connection in the normal bundle $\nu$, we have covariant differential $\nabla$ which maps a section of $v \otimes\left(T^{\star} \Sigma\right)^{k}$ into a section of $v \otimes\left(T^{\star} \Sigma\right)^{k+1}$. In particular, for a normal section of $\Sigma, x=x_{\mu} e_{\mu}$, we define

$$
\nabla x=x_{\mu j} \theta_{j} \otimes e_{\mu},
$$

where $x_{\mu j}$ is the covariant derivative of $x_{\mu}$ and is determined by

$$
x_{\mu j} \theta_{j}=d x_{\mu}+x_{\lambda} \theta_{\lambda \mu} .
$$

Similarly, $x_{\mu j k}$ is the second covariant derivative of $x_{\mu}$ and is determined by

$$
x_{\mu j k} \theta_{k}=d x_{\mu j}+x_{\mu k} \theta_{k j}-x_{\lambda j} \theta_{\mu \lambda} .
$$

Taking the exterior derivative of (2.6), using (2.3), (2.7) and the fact that $\theta_{3}=\theta_{4}=0$ on $\Sigma$, we have

$$
x_{\lambda} d \theta_{\lambda \mu}=x_{\mu j k} \theta_{k} \wedge \theta_{j} .
$$


By (2.2), we see that the curvature of the normal bundle $K_{3412}$ satisfies

$$
d \theta_{34}=-K_{3412} \theta_{1} \wedge \theta_{2} .
$$

By (2.8) and (2.9), we obtain the following identities

$$
-x_{3} K_{3412}=x_{421}-x_{412}
$$

and

$$
x_{4} K_{3412}=x_{321}-x_{312} .
$$

Denote the complexification of $v$ by $v_{\mathbb{C}}=v \otimes \mathbb{C}=\{v+i v\}$. Extend the covariant differential $\nabla$ to $v_{\mathbb{C}}$ complex linearly, i.e., $\nabla(x+i y)=\nabla x+i \nabla y, x$ and $y$ are sections of $\nu$. The surface $\Sigma$ admits a complex structure defined by the $(1,0)$ form $\phi=\theta_{1}+i \theta_{2}$ and the $(0,1)$ form $\bar{\phi}=\theta_{1}-i \theta_{2}$. We can decompose its $(1,0)$ and $(0,1)$ parts and write it as

$$
\nabla=\nabla^{(1,0)}+\nabla^{(0,1)}
$$

Using the Newlander-Nirenberg theorem, $v_{\mathbb{C}}$ can be made into a holomorphic bundle whose $\bar{\partial}$-operator is $\nabla^{(0,1)}$. Let $x=x_{\mu} e_{\mu}$ and $y=y_{\mu} e_{\mu}$ be sections of the normal bundle so that $x+i y$ is a section of $v_{\mathbb{C}}$, we have

$$
\begin{aligned}
\nabla^{(1,0)}(x+i y)= & \frac{1}{2}\left\{\left(x_{31}+y_{32}\right)+i\left(y_{31}-x_{32}\right)\right\} \phi \otimes e_{3} \\
& +\frac{1}{2}\left\{\left(x_{41}+y_{42}\right)+i\left(y_{41}-x_{42}\right)\right\} \phi \otimes e_{4}, \\
\nabla^{(0,1)}(x+i y)= & \frac{1}{2}\left\{\left(x_{31}-y_{32}\right)+i\left(y_{31}+x_{32}\right)\right\} \bar{\phi} \otimes e_{3} \\
& +\frac{1}{2}\left\{\left(x_{41}-y_{42}\right)+i\left(y_{41}+x_{42}\right)\right\} \bar{\phi} \otimes e_{4} \\
= & \bar{\partial}(x+i y) .
\end{aligned}
$$

It is clear that $x+i y$ is a holomorphic section of $\nu_{\mathbb{C}}$ if $\nabla^{(0,1)}(x+i y)=0$.

There exists a natural almost complex structure on $v$, defined by

$$
J_{v}\left(e_{3}\right)=e_{4}, \quad J_{v}\left(e_{4}\right)=-e_{3}
$$

Extend $J_{v}$ to $v_{\mathbb{C}}$ complex linearly. Let $e^{\prime}=\frac{1}{2}\left(e_{3}-i e_{4}\right)$ and $e^{\prime \prime}=\frac{1}{2}\left(e_{3}+i e_{4}\right)$, then

$$
v_{\mathbb{C}}=v^{\prime} \oplus v^{\prime \prime}
$$

where $v^{\prime}$ is spanned by $e^{\prime}, v^{\prime \prime}$ is spanned by $e^{\prime \prime}$ and they are complex line bundles. Notice that

$$
\nabla e^{\prime}=i \theta_{34} \otimes e^{\prime}, \quad \nabla e^{\prime \prime}=-i \theta_{34} \otimes e^{\prime \prime},
$$


$v^{\prime}$ and $v^{\prime \prime}$ are parallel and hence holomorphic sub-bundles of $v_{\mathbb{C}}$. By (2.12), we know that a section $x^{\prime} e^{\prime}$ of $v^{\prime}$ is holomorphic if

$$
\nabla x^{\prime} \equiv 0 \quad \bmod \quad \phi
$$

A normal vector $x=x_{3} e_{3}+x_{4} e_{4}$ can be expressed by $e^{\prime}, e^{\prime \prime}$ uniquely as

$$
\begin{aligned}
x & =\left(x_{3}+i x_{4}\right) e^{\prime}+\left(x_{3}-i x_{4}\right) e^{\prime \prime} \\
& =x^{\prime} e^{\prime}+x^{\prime \prime} e^{\prime \prime} .
\end{aligned}
$$

We identify $x$ with $x^{\prime} e^{\prime}$ and this isomorphism imposes a holomorphic structure on $v$. More precisely, if $x=x_{3} e_{3}+x_{4} e_{4}$ we have

$$
\begin{aligned}
& d\left(x_{3}+i x_{4}\right)+\left(x_{3}+i x_{4}\right) i \theta_{34} \\
& \quad=\frac{1}{2}\left[\left(x_{31}+x_{42}\right)+i\left(x_{41}-x_{32}\right)\right] \phi+\frac{1}{2}\left[\left(x_{31}-x_{42}\right)+i\left(x_{41}+x_{32}\right)\right] \bar{\phi} .
\end{aligned}
$$

Set

$$
\bar{\partial} x=\left(x_{31}-x_{42}\right) \bar{\phi} \otimes e_{3}+\left(x_{41}+x_{32}\right) \bar{\phi} \otimes e_{4},
$$

then $x$ is a holomorphic section of $v$ if and only if $\bar{\partial} x=0$.

\section{Second Variation Formulae}

In this section, we deduce the second variation of the $L$-functional of a symplectic critical surface. Recall that

$$
L(\phi)=\int_{\Sigma} \frac{1}{\cos \alpha} d \mu_{\Sigma},
$$

where $\phi: \Sigma \rightarrow M$ is a smooth symplectic immersion. Let $\phi(\Sigma)$ be a symplectic critical surface of $M$. Let

$$
\phi_{t, \varepsilon}: \Sigma \times(-\delta, \delta) \times(-a, a) \rightarrow M
$$

be one family of smooth immersions with

$$
\phi_{0,0}=\phi
$$

Since symplectic is an open condition, we can assume that $\phi_{t, \varepsilon}(\Sigma)$ is symplectic for every $t \in(-\delta, \delta)$ and every $\varepsilon \in(-a, a)$, which means $\phi_{t, \varepsilon}$ is a symplectic variation of $\phi$. For simplicity, we write $\phi_{0,0}(\Sigma)=\phi(\Sigma)=\Sigma$. Let 


$$
\left.\frac{\partial \phi_{t, 0}}{\partial t}\right|_{t=0}=X,\left.\quad \frac{\partial \phi_{0, \varepsilon}}{\partial \varepsilon}\right|_{\varepsilon=0}=Y \quad \text { and }\left.\quad \frac{\partial^{2} \phi_{t, \varepsilon}}{\partial t \partial \varepsilon}\right|_{t=0, \varepsilon=0}=Z
$$

We assume in this section that $X, Y$ and $Z$ are of compact supports.

Lemma 3.1 We have the general second variation formula:

$$
\begin{aligned}
& \left.\frac{\partial^{2}}{\partial t \partial \varepsilon}\right|_{t=0, \varepsilon=0} L\left(\phi_{t, \varepsilon}\right)=4 \int_{\Sigma} \frac{d i v_{\Sigma} X d i v_{\Sigma} Y}{\cos \alpha}-2 \int_{\Sigma} \frac{R\left(X, e_{i}, Y, e_{i}\right)}{\cos \alpha} d \mu_{\Sigma} \\
& \quad+2 \int_{\Sigma} \frac{\left\langle\bar{\nabla}_{e_{i}}^{\perp} X, \bar{\nabla}_{e_{i}}^{\perp} Y\right\rangle}{\cos \alpha} d \mu_{\Sigma} \\
& \quad-\int_{\Sigma} \frac{\left\langle e_{i}, \bar{\nabla}_{e_{j}} X\right\rangle\left\langle e_{j}, \bar{\nabla}_{e_{i}} Y\right\rangle+\left\langle e_{j}, \bar{\nabla}_{e_{i}} X\right\rangle\left\langle e_{i}, \bar{\nabla}_{e_{j}} Y\right\rangle}{\cos \alpha} d \mu_{\Sigma} \\
& -2 \int_{\Sigma} \frac{d i v_{\Sigma} X\left(\omega\left(\bar{\nabla}_{e_{1}} Y, e_{2}\right)+\omega\left(e_{1}, \bar{\nabla}_{e_{2}} Y\right)\right)}{\cos ^{2} \alpha} d \mu_{\Sigma} \\
& -2 \int_{\Sigma} \frac{d i v_{\Sigma} Y\left(\omega\left(\bar{\nabla}_{e_{1}} X, e_{2}\right)+\omega\left(e_{1}, \bar{\nabla}_{e_{2}} X\right)\right)}{\cos ^{2} \alpha} d \mu_{\Sigma} \\
& -\int_{\Sigma} \frac{\omega\left(R\left(Y, e_{1}\right) X, e_{2}\right)+\omega\left(e_{1}, R\left(Y, e_{2}\right) X\right)}{\cos ^{2} \alpha} d \mu_{\Sigma} \\
& -\int_{\Sigma} \frac{\omega\left(\bar{\nabla}_{e_{1}} X, \bar{\nabla}_{e_{2}} Y\right)+\omega\left(\bar{\nabla}_{e_{1}} Y, \bar{\nabla}_{e_{2}} X\right)}{\cos ^{2} \alpha} d \mu_{\Sigma} \\
& +2 \int_{\Sigma} \frac{\left(\omega\left(\bar{\nabla}_{e_{1}} X, e_{2}\right)+\omega\left(e_{1}, \bar{\nabla}_{e_{2}} X\right)\right)\left(\omega\left(\bar{\nabla}_{e_{1}} Y, e_{2}\right)+\omega\left(e_{1}, \bar{\nabla}_{e_{2}} Y\right)\right)}{\cos ^{3} \alpha} d \mu_{\Sigma},
\end{aligned}
$$

where $\left\{e_{1}, e_{2}\right\}$ is a base of $\Sigma, \bar{\nabla}$ is the covariant differential of $M$, and $\operatorname{div}_{\Sigma} X=$ $\left\langle\bar{\nabla}_{e_{i}} X, e_{i}\right\rangle, R$ is the curvature operator on $M$.

Proof Let $\left(x_{1}, x_{2}\right)$ be a local normal coordinates in $\Sigma$. The induced metric on $\phi_{t, \varepsilon}(\Sigma)$ is

$$
g_{i j}(t, \varepsilon)=\left\langle\frac{\partial \phi_{t, \varepsilon}}{\partial x_{i}}, \frac{\partial \phi_{t, \varepsilon}}{\partial x_{j}}\right\rangle
$$

For simplicity, we denote $\frac{\partial \phi_{0,0}}{\partial x_{i}}$ by $e_{i}, g_{i j}(t, \varepsilon)$ by $g_{i j}$, and $\phi_{t, \varepsilon}$ by $\phi$.

A simple calculation gives

$$
\begin{gathered}
\left.\frac{\partial}{\partial t}\right|_{t=0, \varepsilon=0} g_{i j}=\left\langle\bar{\nabla}_{e_{i}} X, e_{j}\right\rangle+\left\langle e_{i}, \bar{\nabla}_{e_{j}} X\right\rangle, \\
\left.\frac{\partial}{\partial \varepsilon}\right|_{t=0, \varepsilon=0} g_{i j}=\left\langle\bar{\nabla}_{e_{i}} Y, e_{j}\right\rangle+\left\langle e_{i}, \bar{\nabla}_{e_{j}} Y\right\rangle
\end{gathered}
$$


and

$$
\begin{aligned}
\left.\frac{\partial^{2}}{\partial t \partial \varepsilon}\right|_{t=0, \varepsilon=0} g_{i j}= & \left\langle\bar{\nabla}_{e_{i}} Z+R\left(Y, e_{i}\right) X, e_{j}\right\rangle+\left\langle e_{i}, \bar{\nabla}_{e_{j}} Z+R\left(Y, e_{j}\right) X\right\rangle \\
& +\left\langle\bar{\nabla}_{e_{i}} X, \bar{\nabla}_{e_{j}} Y\right\rangle+\left\langle\bar{\nabla}_{e_{i}} Y, \bar{\nabla}_{e_{j}} X\right\rangle .
\end{aligned}
$$

Since

$$
\cos \alpha(\varepsilon, t)=\frac{\omega\left(\frac{\partial \phi}{\partial x_{1}}, \frac{\partial \phi}{\partial x_{2}}\right)}{\sqrt{\operatorname{det}\left(g_{i j}\right)}},
$$

we have

$$
L=\int_{\Sigma} \frac{\operatorname{det}\left(g_{i j}\right)}{\omega\left(\frac{\partial \phi}{\partial x_{1}}, \frac{\partial \phi}{\partial x_{2}}\right)} d x_{1} \wedge d x_{2} .
$$

We write the integrand of the functional $L$ as

$$
I=\frac{\operatorname{det}\left(g_{i j}\right)}{\omega\left(\frac{\partial \phi}{\partial x_{1}}, \frac{\partial \phi}{\partial x_{2}}\right)},
$$

then

$$
\begin{aligned}
\left.\frac{\partial I}{\partial t}\right|_{t=0}= & \frac{\left.\frac{\partial}{\partial t}\right|_{t=0} g_{i j} g^{i j}}{\omega\left(\frac{\partial \phi}{\partial x_{1}}, \frac{\partial \phi}{\partial x_{2}}\right)} \operatorname{det}\left(g_{i j}\right) \\
& -\frac{\omega\left(\left.\bar{\nabla}_{e_{1}} \frac{\partial \phi}{\partial t}\right|_{t=0}, \frac{\partial \phi}{\partial x_{2}}\right)+\omega\left(\frac{\partial \phi}{\partial x_{1}},\left.\bar{\nabla}_{e_{2}} \frac{\partial \phi}{\partial t}\right|_{t=0}\right)}{\omega^{2}\left(\frac{\partial \phi}{\partial x_{1}}, \frac{\partial \phi}{\partial x_{2}}\right)} \operatorname{det}\left(g_{i j}\right) .
\end{aligned}
$$

Thus,

$$
\begin{aligned}
\left.\frac{\partial^{2} I}{\partial t \partial \varepsilon}\right|_{t=0, \varepsilon=0}= & \frac{\left.\left.\frac{\partial}{\partial \varepsilon}\right|_{\varepsilon=0} g_{k l} g^{k l} \frac{\partial}{\partial t}\right|_{t=0} g_{i j} g^{i j}}{\omega\left(e_{1}, e_{2}\right)}+\frac{\left.\frac{\partial^{2}}{\partial \varepsilon \partial t}\right|_{t=0, \varepsilon=0} g_{i j} g^{i j}}{\omega\left(e_{1}, e_{2}\right)}+\frac{\left.\left.\frac{\partial}{\partial t}\right|_{t=0} g_{i j} \frac{\partial}{\partial \varepsilon}\right|_{\varepsilon=0} g^{i j}}{\omega\left(e_{1}, e_{2}\right)} \\
& -\frac{\left.\frac{\partial}{\partial t}\right|_{t=0} g_{i j} g^{i j}\left[\omega\left(\left.\bar{\nabla}_{e_{1}} \frac{\partial \phi}{\partial \varepsilon}\right|_{\varepsilon=0}, e_{2}\right)+\omega\left(e_{1},\left.\bar{\nabla}_{e_{2}} \frac{\partial \phi}{\partial \varepsilon}\right|_{\varepsilon=0}\right)\right]}{\omega^{2}\left(e_{1}, e_{2}\right)} \\
& -\frac{\left.\frac{\partial}{\partial \varepsilon}\right|_{\varepsilon=0} g_{i j} g^{i j}\left[\omega\left(\left.\bar{\nabla}_{e_{1}} \frac{\partial \phi}{\partial t}\right|_{t=0}, e_{2}\right)+\omega\left(e_{1},\left.\bar{\nabla}_{e_{2}} \frac{\partial \phi}{\partial t}\right|_{t=0}\right)\right]}{\omega^{2}\left(e_{1}, e_{2}\right)} \\
& -\frac{\omega\left(\left.\bar{\nabla}_{e_{1}} \frac{\partial \phi}{\partial t}\right|_{t=0},\left.\bar{\nabla}_{e_{2}} \frac{\partial \phi}{\partial \varepsilon}\right|_{\varepsilon=0}\right)+\omega\left(\left.\bar{\nabla}_{e_{1}} \frac{\partial \phi}{\partial \varepsilon}\right|_{\varepsilon=0},\left.\bar{\nabla}_{e_{2}} \frac{\partial \phi}{\partial t}\right|_{t=0}\right)}{\omega^{2}\left(e_{1}, e_{2}\right)} \\
& -\frac{\omega\left(\left.\bar{\nabla}_{e_{1}} \frac{\partial^{2} \phi}{\partial t \partial \varepsilon}\right|_{t=0, \varepsilon=0}+\left.R\left(Y, e_{1}\right) \frac{\partial \phi}{\partial t}\right|_{t=0}, e_{2}\right)}{\omega^{2}\left(e_{1}, e_{2}\right)}
\end{aligned}
$$




$$
\begin{aligned}
& -\frac{\omega\left(e_{1},\left.\bar{\nabla}_{e_{2}} \frac{\partial^{2} \phi}{\partial t \partial \varepsilon}\right|_{t=0, \varepsilon=0}+\left.R\left(Y, e_{2}\right) \frac{\partial \phi}{\partial t}\right|_{t=0}\right)}{\omega^{2}\left(e_{1}, e_{2}\right)} \\
& +\frac{2}{\omega^{3}\left(e_{1}, e_{2}\right)}\left[\omega\left(\left.\bar{\nabla}_{e_{1}} \frac{\partial \phi}{\partial t}\right|_{t=0}, \frac{\partial \phi}{\partial x_{2}}\right)+\omega\left(\frac{\partial \phi}{\partial x_{1}},\left.\bar{\nabla}_{e_{2}} \frac{\partial \phi}{\partial t}\right|_{t=0}\right)\right] \\
& \cdot\left[\omega\left(\left.\bar{\nabla}_{e_{1}} \frac{\partial \phi}{\partial \varepsilon}\right|_{\varepsilon=0}, \frac{\partial \phi}{\partial x_{2}}\right)+\omega\left(\frac{\partial \phi}{\partial x_{1}},\left.\bar{\nabla}_{e_{2}} \frac{\partial \phi}{\partial \varepsilon}\right|_{\varepsilon=0}\right)\right] .
\end{aligned}
$$

By (3.2)-(3.4), we get that

$$
\begin{aligned}
\left.\frac{\partial^{2} I}{\partial t \partial \varepsilon}\right|_{t=0, \varepsilon=0}= & 4 \frac{\left\langle\bar{\nabla}_{e_{j}} X, e_{j}\right\rangle\left\langle\bar{\nabla}_{e_{i}} Y, e_{i}\right\rangle}{\omega\left(e_{1}, e_{2}\right)}+2 \frac{\left\langle\bar{\nabla}_{e_{i}} Z, e_{i}\right\rangle}{\omega\left(e_{1}, e_{2}\right)} \\
& +2 \frac{\left\langle\bar{\nabla}_{e_{i}} X, \bar{\nabla}_{e_{i}} Y\right\rangle}{\omega\left(e_{1}, e_{2}\right)}-2 \frac{R\left(X, e_{i}, Y, e_{i}\right)}{\omega\left(e_{1}, e_{2}\right)} \\
& -\frac{\left(\left\langle\bar{\nabla}_{e_{i}} X, e_{j}\right\rangle+\left\langle e_{i}, \bar{\nabla}_{e_{j}} X\right\rangle\right)\left(\left\langle\bar{\nabla}_{e_{i}} Y, e_{j}\right\rangle+\left\langle e_{i}, \bar{\nabla}_{e_{j}} Y\right\rangle\right)}{\omega\left(e_{1}, e_{2}\right)} \\
& -2 \frac{\left\langle\bar{\nabla}_{e_{i}} X, e_{i}\right\rangle\left[\omega\left(\bar{\nabla}_{e_{1}} Y, e_{2}\right)+\omega\left(e_{1}, \bar{\nabla}_{e_{2}} Y\right)\right]}{\omega^{2}\left(e_{1}, e_{2}\right)} \\
& -2 \frac{\left\langle\bar{\nabla}_{e_{i}} Y, e_{i}\right\rangle\left[\omega\left(\bar{\nabla}_{e_{1}} X, e_{2}\right)+\omega\left(e_{1}, \bar{\nabla}_{e_{2}} X\right)\right]}{\omega^{2}\left(e_{1}, e_{2}\right)} \\
& -\frac{\omega\left(\bar{\nabla}_{e_{1}} Z, e_{2}\right)+\omega\left(e_{1}, \bar{\nabla}_{e_{2}} Z\right)}{\omega^{2}\left(e_{1}, e_{2}\right)} \\
& -\frac{\omega\left(R\left(Y, e_{1}\right) X, e_{2}\right)+\omega\left(e_{1}, R\left(Y, e_{2}\right) X\right)}{\omega \omega^{2}\left(e_{1}, e_{2}\right)} \\
& -\frac{\omega\left(\bar{\nabla}_{e_{1}} X, \bar{\nabla}_{e_{2}} Y\right)+\omega\left(\bar{\nabla}_{e_{1}} Y, \bar{\nabla}_{e_{2}} X\right)}{\omega^{2}\left(e_{1}, e_{2}\right)} \\
& +2 \frac{\left[\omega\left(\bar{\nabla}_{e_{1}} X, e_{2}\right)+\omega\left(e_{1}, \bar{\nabla}_{e_{2}} X\right)\right]\left[\omega\left(\bar{\nabla}_{e_{1}} Y, e_{2}\right)+\omega\left(e_{1}, \bar{\nabla}_{e_{2}} Y\right)\right]}{\omega} .
\end{aligned}
$$

Since $\Sigma$ is a symplectic critical surface, by the first variation formula [3], we have

$$
\int_{\Sigma}\left(\frac{2\left\langle\bar{\nabla}_{e_{i}} Z, e_{i}\right\rangle}{\cos \alpha}-\frac{\omega\left(\bar{\nabla}_{e_{1}} Z, e_{2}\right)+\omega\left(e_{1}, \bar{\nabla}_{e_{2}} Z\right)}{\cos ^{2} \alpha}\right) d \mu_{\Sigma}=0
$$

Notice that

$$
\begin{array}{r}
2\left\langle\bar{\nabla}_{e_{i}} X, \bar{\nabla}_{e_{i}} Y\right\rangle-\left(\left\langle\bar{\nabla}_{e_{i}} X, e_{j}\right\rangle+\left\langle e_{i}, \bar{\nabla}_{e_{j}} X\right\rangle\right)\left(\left\langle\bar{\nabla}_{e_{i}} Y, e_{j}\right\rangle+\left\langle e_{i}, \bar{\nabla}_{e_{j}} Y\right\rangle\right) \\
=2\left\langle\bar{\nabla}_{e_{i}}^{\perp} X, \bar{\nabla}_{e_{i}}^{\perp} Y\right\rangle-\left(\left\langle e_{i}, \bar{\nabla}_{e_{j}} X\right\rangle\left\langle e_{j}, \bar{\nabla}_{e_{i}} Y\right\rangle+\left\langle e_{i}, \bar{\nabla}_{e_{j}} Y\right\rangle\left\langle e_{j}, \bar{\nabla}_{e_{i}} X\right\rangle\right)
\end{array}
$$


and

$$
\left\langle\bar{\nabla}_{e_{i}} X, e_{i}\right\rangle=\operatorname{div} v_{\Sigma} X
$$

Putting (3.7)-(3.9) into (3.6), we obtain the identity in the lemma.

If $X=Y$ is a normal variation, we have

$$
\begin{aligned}
& \operatorname{div}_{\Sigma} X=-X \cdot H, \\
& \left\langle e_{i}, \bar{\nabla}_{e_{j}} X\right\rangle\left\langle e_{j}, \bar{\nabla}_{e_{i}} X\right\rangle=\left(X \cdot A\left(e_{i}, e_{j}\right)\right)^{2}
\end{aligned}
$$

and

$$
\begin{aligned}
- & \int_{\Sigma} \frac{\omega\left(R\left(X, e_{1}\right) X, e_{2}\right)+\omega\left(e_{1}, R\left(X, e_{2}\right) X\right)}{\cos ^{2} \alpha} d \mu_{\Sigma}-2 \int_{\Sigma} \frac{\omega\left(\bar{\nabla}_{e_{1}} X, \bar{\nabla}_{e_{2}} X\right)}{\cos ^{2} \alpha} d \mu_{\Sigma} \\
= & \int_{\Sigma} \frac{\left\langle J e_{2}, R\left(X, e_{1}\right) X\right\rangle-\left\langle J e_{1}, R\left(X, e_{2}\right) X\right\rangle}{\cos ^{2} \alpha} d \mu_{\Sigma} \\
& -\int_{\Sigma} \frac{\bar{\nabla}_{e_{1}}\left(\omega\left(X, \bar{\nabla}_{e_{2}} X\right)\right)-\omega\left(X, \bar{\nabla}_{e_{1}} \bar{\nabla}_{e_{2}} X\right)}{\cos ^{2} \alpha} d \mu_{\Sigma} \\
& -\int_{\Sigma} \frac{\bar{\nabla}_{e_{2}}\left(\omega\left(\bar{\nabla}_{e_{1}} X, X\right)\right)-\omega\left(\bar{\nabla}_{e_{2}} \bar{\nabla}_{e_{1}} X, X\right)}{\cos ^{2} \alpha} d \mu_{\Sigma} \\
= & \int_{\Sigma} \frac{R\left(X, e_{1}, J e_{2}, X\right)+R\left(X, e_{2}, X, J e_{1}\right)+R\left(e_{1}, e_{2}, J X, X\right)}{\cos ^{2} \alpha} d \mu_{\Sigma} \\
& -2 \int_{\Sigma} \frac{\omega\left(X, \bar{\nabla}_{e_{2}} X\right) \nabla_{e_{1}} \cos \alpha+\omega\left(\bar{\nabla}_{e_{1}} X, X\right) \nabla_{e_{2}} \cos \alpha}{\cos ^{3} \alpha} d \mu_{\Sigma},
\end{aligned}
$$

in the last step, we used integration by parts.

By the Bianchi equality, we have

$$
\begin{aligned}
& R\left(X, e_{1}, J e_{2}, X\right)+R\left(X, e_{2}, X, J e_{1}\right)+R\left(e_{1}, e_{2}, J X, X\right) \\
& \quad=R\left(X, e_{1}, J X, e_{2}\right)+R\left(X, e_{2}, e_{1}, J X\right)+R\left(X, J X, e_{2}, e_{1}\right)=0 .
\end{aligned}
$$

Therefore, by (3.10)-(3.13), we obtain the following formula.

Corollary 3.2 If $X$ is a normal variation with compact support, then

$$
\begin{aligned}
\left.\frac{\partial^{2}}{\partial t^{2}}\right|_{t=0} L\left(\phi_{t}\right)= & : I I(X) \\
= & 4 \int_{\Sigma} \frac{(X \cdot H)^{2}}{\cos \alpha} d \mu_{\Sigma}-2 \int_{\Sigma} \frac{R\left(X, e_{i}, X, e_{i}\right)}{\cos \alpha} d \mu_{\Sigma} \\
& +2 \int_{\Sigma} \frac{\left|\bar{\nabla}_{e_{i}}^{\perp} X\right|^{2}}{\cos \alpha} d \mu_{\Sigma}-2 \int_{\Sigma} \frac{\left(X \cdot A\left(e_{i}, e_{j}\right)\right)^{2}}{\cos \alpha} d \mu_{\Sigma} \\
& +4 \int_{\Sigma} \frac{X \cdot H\left(\omega\left(\bar{\nabla}_{e_{1}} X, e_{2}\right)+\omega\left(e_{1}, \bar{\nabla}_{e_{2}} X\right)\right)}{\cos ^{2} \alpha} d \mu_{\Sigma}
\end{aligned}
$$




$$
\begin{aligned}
& +2 \int_{\Sigma} \frac{\left[\omega\left(\bar{\nabla}_{e_{1}} X, e_{2}\right)+\omega\left(e_{1}, \bar{\nabla}_{e_{2}} X\right)\right]^{2}}{\cos ^{3} \alpha} d \mu_{\Sigma} \\
& -2 \int_{\Sigma} \frac{\omega\left(X, \bar{\nabla}_{e_{2}} X\right) \nabla_{e_{1}} \cos \alpha+\omega\left(\bar{\nabla}_{e_{1}} X, X\right) \nabla_{e_{2}} \cos \alpha}{\cos ^{3} \alpha} d \mu_{\Sigma} .
\end{aligned}
$$

Set $X=x_{3} e_{3}+x_{4} e_{4}$, then

$$
\begin{aligned}
\omega\left(X, \bar{\nabla}_{e_{2}} X\right)= & \left\langle J X, \bar{\nabla}_{e_{2}} X\right\rangle \\
= & \left\langle x_{3} J e_{3}+x_{4} J e_{4}, x_{32} e_{3}+x_{42} e_{4}-x_{3} h_{2 i}^{3} e_{i}-x_{4} h_{2 l}^{4} e_{l}\right\rangle \\
= & x_{3} x_{42}\left\langle J e_{3}, e_{4}\right\rangle+x_{4} x_{32}\left\langle J e_{4}, e_{3}\right\rangle \\
& +x_{3}\left(x_{3} h_{12}^{3}+x_{4} h_{12}^{4}\right)\left\langle e_{3}, J e_{1}\right\rangle+x_{3}\left(x_{3} h_{22}^{3}+x_{4} h_{22}^{4}\right)\left\langle e_{3}, J e_{2}\right\rangle \\
& +x_{4}\left(x_{3} h_{12}^{3}+x_{4} h_{12}^{4}\right)\left\langle e_{4}, J e_{1}\right\rangle+x_{4}\left(x_{3} h_{22}^{3}+x_{4} h_{22}^{4}\right)\left\langle e_{4}, J e_{2}\right\rangle .
\end{aligned}
$$

Notice that

$$
\begin{aligned}
& \left\langle J e_{1}, e_{2}\right\rangle=\left\langle J e_{3}, e_{4}\right\rangle=\cos \alpha, \\
& \left\langle J e_{1}, e_{3}\right\rangle=-\left\langle J e_{2}, e_{4}\right\rangle, \\
& \left\langle J e_{1}, e_{4}\right\rangle=\left\langle J e_{2}, e_{3}\right\rangle .
\end{aligned}
$$

Thus

$$
\begin{aligned}
\omega\left(X, \bar{\nabla}_{e_{2}} X\right)= & \left(x_{3} x_{42}-x_{4} x_{32}\right) \cos \alpha \\
& +\left(x_{3}^{2} h_{12}^{3}+x_{3} x_{4} h_{12}^{4}-x_{3} x_{4} h_{22}^{3}-x_{4}^{2} h_{22}^{4}\right)\left\langle e_{3}, J e_{1}\right\rangle \\
& +\left(x_{3}^{2} h_{22}^{3}+x_{3} x_{4} h_{22}^{4}+x_{3} x_{4} h_{12}^{3}+x_{4}^{2} h_{12}^{4}\right)\left\langle e_{3}, J e_{2}\right\rangle
\end{aligned}
$$

and

$$
\begin{aligned}
\omega\left(X, \bar{\nabla}_{e_{1}} X\right)= & \left(x_{3} x_{41}-x_{4} x_{31}\right) \cos \alpha \\
& +\left(x_{3}^{2} h_{11}^{3}+x_{3} x_{4} h_{11}^{4}-x_{3} x_{4} h_{12}^{3}-x_{4}^{2} h_{12}^{4}\right)\left\langle e_{3}, J e_{1}\right\rangle \\
& +\left(x_{3}^{2} h_{12}^{3}+x_{3} x_{4} h_{12}^{4}+x_{3} x_{4} h_{11}^{3}+x_{4}^{2} h_{11}^{4}\right)\left\langle e_{3}, J e_{2}\right\rangle .
\end{aligned}
$$

Similarly,

$$
\begin{aligned}
\omega\left(\bar{\nabla}_{e_{1}} X, e_{2}\right)+\omega\left(e_{1}, \bar{\nabla}_{e_{2}} X\right)= & -\cos \alpha\left(x_{3} H^{3}+x_{4} H^{4}\right) \\
& +\left(x_{32}+x_{41}\right)\left\langle J e_{1}, e_{3}\right\rangle+\left(x_{42}-x_{31}\right)\left\langle J e_{2}, e_{3}\right\rangle .
\end{aligned}
$$

By (3.20), we get that

$$
\begin{aligned}
& 4 \int_{\Sigma} \frac{X \cdot H\left(\omega\left(\bar{\nabla}_{e_{1}} X, e_{2}\right)+\omega\left(e_{1}, \bar{\nabla}_{e_{2}} X\right)\right)}{\cos ^{2} \alpha}+2 \int_{\Sigma} \frac{\left[\omega\left(\bar{\nabla}_{e_{1}} X, e_{2}\right)+\omega\left(e_{1}, \bar{\nabla}_{e_{2}} X\right)\right]^{2}}{\cos ^{3} \alpha} \\
& =-2 \int_{\Sigma} \frac{\left(x_{3} H^{3}+x_{4} H^{4}\right)^{2}}{\cos \alpha} d \mu_{\Sigma}+2 \int_{\Sigma} \frac{\left(x_{31}+x_{41}\right)^{2}\left\langle J e_{1}, e_{3}\right\rangle^{2}}{\cos ^{3} \alpha} d \mu_{\Sigma} \\
& \quad+2 \int_{\Sigma} \frac{\left(x_{42}-x_{31}\right)^{2}\left\langle J e_{2}, e_{3}\right\rangle^{2}}{\cos ^{3} \alpha} d \mu_{\Sigma} \\
& \quad+4 \int_{\Sigma} \frac{\left(x_{32}+x_{41}\right)\left(x_{42}-x_{31}\right)\left\langle J e_{1}, e_{3}\right\rangle\left\langle J e_{2}, e_{3}\right\rangle}{\cos ^{3} \alpha} d \mu_{\Sigma} .
\end{aligned}
$$


Now $I I(X)$ can be written as

$$
\begin{aligned}
I I(X)= & -2 \int_{\Sigma} \frac{x_{3}^{2} R_{3 i 3 i}+x_{4}^{2} R_{4 i 4 i}+2 x_{3} x_{4} R_{3 i 4 i}}{\cos \alpha} d \mu_{\Sigma}+2 \int_{\Sigma} \frac{x_{3 i}^{2}+x_{4 i}^{2}}{\cos \alpha} \\
& -4 \int_{\Sigma} \frac{\left(x_{3} h_{12}^{3}+x_{4} h_{12}^{4}\right)^{2}-\left(x_{3} h_{11}^{3}+x_{4} h_{11}^{4}\right)\left(x_{3} h_{22}^{3}+x_{4} h_{22}^{4}\right)}{\cos \alpha} \\
& +2 \int_{\Sigma} \frac{\left(x_{32}+x_{41}\right)^{2}\left\langle J e_{1}, e_{3}\right\rangle^{2}+\left(x_{42}-x_{31}\right)^{2}\left\langle J e_{2}, e_{3}\right\rangle^{2}}{\cos ^{3} \alpha} \\
& +4 \int_{\Sigma} \frac{\left(x_{32}+x_{41}\right)\left(x_{42}-x_{31}\right)\left\langle J e_{1}, e_{3}\right\rangle\left\langle J e_{2}, e_{3}\right\rangle}{\cos ^{3} \alpha} d \mu_{\Sigma} \\
& +I I I(X),
\end{aligned}
$$

where

$$
I I I(X)=-2 \int_{\Sigma} \frac{\omega\left(X, \bar{\nabla}_{e_{2}} X\right) \nabla_{e_{1}} \cos \alpha+\omega\left(\bar{\nabla}_{e_{1}} X, X\right) \nabla_{e_{2}} \cos \alpha}{\cos ^{3} \alpha} d \mu_{\Sigma}
$$

In addition, if we set $Y=-J_{v} X=x_{4} e_{3}-x_{3} e_{4}$, by (3.22), we get that

$$
\begin{aligned}
I I(X)+I I(Y)= & -2 \int_{\Sigma} \frac{|X|^{2}\left(R_{3 i 3 i}+R_{4 i 4 i}\right)}{\cos \alpha} d \mu_{\Sigma}+4 \int_{\Sigma} \frac{x_{3 i}^{2}+x_{4 i}^{2}}{\cos \alpha} d \mu_{\Sigma} \\
& -4 \int_{\Sigma} \frac{|X|^{2}\left(\left(h_{12}^{3}\right)^{2}+\left(h_{12}^{4}\right)^{2}-h_{11}^{3} h_{22}^{3}-h_{11}^{4} h_{22}^{4}\right)}{\cos \alpha} d \mu_{\Sigma} \\
& +2 \int_{\Sigma} \frac{\left(\left(x_{32}+x_{41}\right)^{2}+\left(x_{42}-x_{31}\right)^{2}\right)\left(\left\langle J e_{1}, e_{3}\right\rangle^{2}+\left\langle J e_{2}, e_{3}\right\rangle^{2}\right)}{\cos ^{3} \alpha} d \mu_{\Sigma} \\
& +I I I(X)+I I I(Y) .
\end{aligned}
$$

Using (3.18) and (3.19), it is easy to check that

$$
\begin{aligned}
I I I(X)+I I I(Y)= & 4 \int_{\Sigma}\left(x_{3} x_{42}-x_{4} x_{32}\right) \nabla_{e_{1}} \frac{1}{\cos \alpha} d \mu_{\Sigma} \\
& -4 \int_{\Sigma}\left(x_{3} x_{41}-x_{4} x_{31}\right) \nabla_{e_{2}} \frac{1}{\cos \alpha} d \mu_{\Sigma} \\
& -2 \int_{\Sigma} \frac{|X|^{2}\left(h_{12}^{3}-h_{22}^{4}\right) \nabla_{e_{1}} \cos \alpha\left\langle e_{3}, J e_{1}\right\rangle}{\cos ^{3} \alpha} d \mu_{\Sigma} \\
& -2 \int_{\Sigma} \frac{|X|^{2}\left(h_{22}^{3}+h_{12}^{4}\right) \nabla_{e_{1}} \cos \alpha\left\langle e_{4}, J e_{1}\right\rangle}{\cos ^{3} \alpha} d \mu_{\Sigma} \\
& +2 \int_{\Sigma} \frac{|X|^{2}\left(h_{11}^{3}-h_{12}^{4}\right) \nabla_{e_{2}} \cos \alpha\left\langle e_{3}, J e_{1}\right\rangle}{\cos ^{3} \alpha} d \mu_{\Sigma} . \\
& +2 \int_{\Sigma} \frac{|X|^{2}\left(h_{12}^{3}+h_{11}^{4}\right) \nabla_{e_{2}} \cos \alpha\left\langle e_{4}, J e_{1}\right\rangle}{\cos ^{3} \alpha} d \mu_{\Sigma} .
\end{aligned}
$$


Integrating the first two terms in (3.24) by parts, we get that

$$
\begin{aligned}
& 4 \int_{\Sigma}\left(x_{3} x_{42}-x_{4} x_{32}\right) \nabla_{e_{1}} \frac{1}{\cos \alpha} d \mu_{\Sigma}-4 \int_{\Sigma}\left(x_{3} x_{41}-x_{4} x_{31}\right) \nabla_{e_{2}} \frac{1}{\cos \alpha} d \mu_{\Sigma} \\
& =4 \int_{\Sigma} \frac{2\left(x_{41} x_{32}-x_{31} x_{42}\right)+\left(x_{4} x_{321}-x_{3} x_{421}\right)+\left(x_{3} x_{412}-x_{4} x_{312}\right)}{\cos \alpha} d \mu_{\Sigma} \\
& =8 \int_{\Sigma} \frac{x_{41} x_{32}-x_{31} x_{42}}{\cos \alpha} d \mu_{\Sigma}+4 \int_{\Sigma} \frac{|X|^{2} K_{3412}}{\cos \alpha} d \mu_{\Sigma},
\end{aligned}
$$

where we have used (2.10) and (2.11).

We compute the last two terms at each point, so we may choose an orthonormal frame $\left\{e_{1}, e_{2}, e_{3}, e_{4}\right\}$ around the point such that $\omega, J$ take the forms (cf. $\left.[1,2]\right)$,

$$
\omega=\cos \alpha \theta_{1} \wedge \theta_{2}+\cos \alpha \theta_{3} \wedge \theta_{4}+\sin \alpha \theta_{1} \wedge \theta_{3}-\sin \alpha \theta_{2} \wedge \theta_{4},
$$

where $\left\{\theta_{1}, \theta_{2}, \theta_{3}, \theta_{4}\right\}$ is the dual frame of $\left\{e_{1}, e_{2}, e_{3}, e_{4}\right\}$, and

$$
J=\left(\begin{array}{cccr}
0 & \cos \alpha & \sin \alpha & 0 \\
-\cos \alpha & 0 & 0 & -\sin \alpha \\
-\sin \alpha & 0 & 0 & \cos \alpha \\
0 & \sin \alpha & -\cos \alpha & 0
\end{array}\right)
$$

By the equation of a symplectic critical surface [3], we have

$$
H^{4}=\frac{\sin ^{2} \alpha}{\cos ^{2} \alpha} \nabla_{e_{1}} \alpha \text {, and } H^{3}=\frac{\sin ^{2} \alpha}{\cos ^{2} \alpha} \nabla_{e_{2}} \alpha \text {. }
$$

Thus

$$
\begin{aligned}
& -2\left(h_{12}^{3}-h_{22}^{4}\right) \nabla_{e_{1}} \cos \alpha+2\left(h_{11}^{3}-h_{12}^{4}\right) \nabla_{e_{2}} \cos \alpha \\
& =2 \sin \alpha\left(h_{12}^{3}+h_{11}^{4}-H^{4}\right) \nabla_{e_{1}} \alpha+2 \sin \alpha\left(h_{12}^{4}+h_{22}^{3}-H^{3}\right) \nabla_{e_{2}} \alpha \\
& =-2 \sin \alpha\left(\nabla_{e_{1}} \alpha+\frac{\sin ^{2} \alpha}{\cos ^{2} \alpha} \nabla_{e_{1}} \alpha\right) \nabla_{e_{1}} \alpha \\
& -2 \sin \alpha\left(\nabla_{e_{2}} \alpha+\frac{\sin ^{2} \alpha}{\cos ^{2} \alpha} \nabla_{e_{2}} \alpha\right) \nabla_{e_{2}} \alpha \\
& =-2 \frac{\sin \alpha}{\cos ^{2} \alpha}|\nabla \alpha|^{2},
\end{aligned}
$$

and

$$
\begin{gathered}
2\left(h_{22}^{3}+h_{12}^{4}\right) \nabla_{e_{1}} \cos \alpha-2\left(h_{12}^{3}+h_{11}^{4}\right) \nabla_{e_{2}} \cos \alpha \\
=2 \sin \alpha \nabla_{e_{2}} \alpha \nabla_{e_{1}} \alpha-2 \sin \alpha \nabla_{e_{2}} \alpha \nabla_{e_{1}} \alpha=0 .
\end{gathered}
$$


By the Ricci equation

$$
K_{3412}=R_{3412}+h_{1 k}^{3} h_{2 k}^{4}-h_{2 k}^{3} h_{1 k}^{4}
$$

we obtain that

$$
\begin{aligned}
I I I(X)+I I I(Y)= & 8 \int_{\Sigma} \frac{x_{41} x_{32}-x_{31} x_{42}}{\cos \alpha} d \mu_{\Sigma}+4 \int_{\Sigma} \frac{|X|^{2} R_{3412}}{\cos \alpha} d \mu_{\Sigma} \\
& -2 \int_{\Sigma} \frac{|X|^{2} \sin ^{2} \alpha}{\cos ^{5} \alpha}|\nabla \alpha|^{2} d \mu_{\Sigma} \\
& +4 \int_{\Sigma} \frac{h_{1 k}^{3} h_{2 k}^{4}-h_{2 k}^{3} h_{1 k}^{4}}{\cos \alpha} d \mu_{\Sigma} .
\end{aligned}
$$

Putting all these identities into (3.23), we obtain that

$$
\begin{aligned}
& I I(X)+I I(Y)= \\
& -2 \int_{\Sigma} \frac{|X|^{2}\left(R_{3 i 3 i}+R_{4 i 4 i}-2 R_{1234}\right)}{\cos \alpha} d \mu_{\Sigma} \\
& +4 \int_{\Sigma} \frac{\left(x_{41}+x_{32}\right)^{2}+\left(x_{42}-x_{31}\right)^{2}}{\cos \alpha} d \mu_{\Sigma} \\
& +2 \int_{\Sigma} \frac{\left[\left(x_{41}+x_{32}\right)^{2}+\left(x_{42}-x_{31}\right)^{2}\right] \sin ^{2} \alpha}{\cos ^{3} \alpha} d \mu_{\Sigma} \\
& -2 \int_{\Sigma} \frac{|X|^{2} \sin ^{2} \alpha}{\cos ^{5} \alpha}|\nabla \alpha|^{2} d \mu_{\Sigma} \\
& -4 \int_{\Sigma} \frac{|X|^{2}\left(\left(h_{12}^{3}\right)^{2}+\left(h_{12}^{4}\right)^{2}-h_{11}^{3} h_{22}^{3}-h_{11}^{4} h_{22}^{4}-h_{1 k}^{3} h_{2 k}^{4}+h_{2 k}^{3} h_{1 k}^{4}\right)}{\cos \alpha} d \mu_{\Sigma} .
\end{aligned}
$$

By Lemma 3.2 in [3], we see that

$$
R_{3 i 3 i}+R_{4 i 4 i}-2 R_{1234}=\frac{R}{2} \sin ^{2} \alpha
$$

By (2.14), we have

$$
\left(x_{41}+x_{32}\right)^{2}+\left(x_{42}-x_{31}\right)^{2}=\frac{1}{2}|\bar{\partial} X|^{2} .
$$


Using the fact that $\Sigma$ is a symplectic critical surface again, we can simplify the last term in the above expression of $I I(X)+I I(Y)$,

$$
\begin{aligned}
& \left(h_{12}^{3}\right)^{2}+\left(h_{12}^{4}\right)^{2}-h_{11}^{3} h_{22}^{3}-h_{11}^{4} h_{22}^{4}-h_{1 k}^{3} h_{2 k}^{4}+h_{2 k}^{3} h_{1 k}^{4} \\
& =\left(h_{11}^{4}+h_{12}^{3}\right)\left(h_{12}^{3}-h_{22}^{4}\right)+\left(h_{12}^{4}+h_{22}^{3}\right)\left(h_{12}^{4}-h_{11}^{3}\right) \\
& =-\nabla_{e_{1}} \alpha\left(-\nabla_{e_{1}} \alpha-H^{4}\right)-\nabla_{e_{2}} \alpha\left(-\nabla_{e_{2}} \alpha-H^{3}\right) \\
& =-\nabla_{e_{1}} \alpha\left(-\nabla_{e_{1}} \alpha-\frac{\sin ^{2} \alpha}{\cos ^{2} \alpha} \nabla_{e_{1}} \alpha\right)-\nabla_{e_{2}} \alpha\left(-\nabla_{e_{2}} \alpha-\frac{\sin ^{2} \alpha}{\cos ^{2} \alpha} \nabla_{e_{2}} \alpha\right) \\
& =\frac{1}{\cos ^{2} \alpha}|\nabla \alpha|^{2} .
\end{aligned}
$$

Therefore, it concludes that

$$
\begin{aligned}
I I(X)+I I(Y)= & -\int_{\Sigma} \frac{R|X|^{2} \sin ^{2} \alpha}{\cos \alpha} d \mu_{\Sigma}+2 \int_{\Sigma} \frac{|\bar{\partial} X|^{2}}{\cos \alpha} d \mu_{\Sigma}+\int_{\Sigma} \frac{\sin ^{2} \alpha}{\cos ^{3} \alpha}|\bar{\partial} X|^{2} d \mu_{\Sigma} \\
& -2 \int_{\Sigma} \frac{\sin ^{2} \alpha}{\cos ^{5} \alpha}|X|^{2}|\nabla \alpha|^{2} d \mu_{\Sigma}-4 \int_{\Sigma} \frac{|X|^{2}|\nabla \alpha|^{2}}{\cos ^{3} \alpha} d \mu_{\Sigma} \\
= & -\int_{\Sigma} \frac{R|X|^{2} \sin ^{2} \alpha}{\cos \alpha} d \mu_{\Sigma}+\int_{\Sigma} \frac{|\bar{\partial} X|^{2}\left(1+\cos ^{2} \alpha\right)}{\cos ^{3} \alpha} d \mu_{\Sigma} \\
& -2 \int_{\Sigma} \frac{\left(1+\cos ^{2} \alpha\right)}{\cos ^{5} \alpha}|X|^{2}|\nabla \alpha|^{2} d \mu_{\Sigma} .
\end{aligned}
$$

Theorem 3.3 If we choose $X=x_{3} e_{3}+x_{4} e_{4}, Y=-J_{v} X=x_{4} e_{3}-x_{3} e_{4}$, which are of compact supports, then the second variation of the functional $L$ of a symplectic critical surface is

$$
\begin{aligned}
I I(X)+I I(Y)= & -\int_{\Sigma} \frac{R|X|^{2} \sin ^{2} \alpha}{\cos \alpha} d \mu_{\Sigma}+\int_{\Sigma} \frac{|\bar{\partial} X|^{2}\left(1+\cos ^{2} \alpha\right)}{\cos ^{3} \alpha} d \mu_{\Sigma} \\
& -2 \int_{\Sigma} \frac{\left(1+\cos ^{2} \alpha\right)}{\cos ^{5} \alpha}|X|^{2}|\nabla \alpha|^{2} d \mu_{\Sigma} .
\end{aligned}
$$

\section{Stable Symplectic Critical Surfaces}

In this section, as an application of the second variation formulae of the functional $L$, we study stable symplectic critical surfaces.

Proposition 4.1 Let $M$ be a complete Kähler surface with positive scalar curvature $R$. If $\Sigma$ is a stable symplectic critical surface in $M$ whose normal bundle admits a section $X$ with

$$
\frac{|\bar{\partial} X|^{2}}{|X|^{2}} \leq \frac{|\nabla \alpha|^{2}}{\cos ^{2} \alpha}
$$


and

$$
\int_{\Sigma} \frac{|X|^{2}}{\cos ^{3} \alpha} d \mu_{\Sigma}<\infty
$$

then $\Sigma$ is a holomorphic curve.

Proof Let $\psi \in C_{0}^{\infty}(M)$ be a cut-off function, with $\psi \equiv 1$ in $B_{r}\left(x_{0}\right), \psi \equiv 0$ outside $B_{2 r}\left(x_{0}\right)$ and $|\nabla \psi| \leq C / r$, where $x_{0} \in \Sigma$. If $\Sigma$ is a stable symplectic critical surface, we have

$$
I I(\psi X)+I I(\psi Y) \geq 0
$$

where $Y=-J_{v} X$. By Theorem 3.3, we obtain

$$
\int_{B_{r}\left(x_{0}\right)} \frac{R|X|^{2} \sin ^{2} \alpha}{\cos \alpha} d \mu_{\Sigma} \leq \frac{C}{r^{2}} .
$$

Since $R>0$, by the last inequality, letting $r \rightarrow \infty$, we obtain $\sin \alpha \equiv 0$, that is $\Sigma$ is a holomorphic curve.

The following corollary is obvious.

Corollary 4.2 Let $M$ be a complete Kähler surface with positive scalar curvature $R$. If $\Sigma$ is a stable symplectic critical surface in $M$ with $\cos \alpha \geq \delta>0$, whose normal bundle admits a holomorphic section $X \in L^{2}(\Sigma)$, then $\Sigma$ is a holomorphic curve.

Corollary 4.3 Let $M$ be a complete Kähler surface with positive scalar curvature $R$. If $\Sigma$ is a compact stable symplectic critical surface in $M$ with $\chi(\nu) \geq g$ where $\chi(v)$ is the Euler characteristic of the normal bundle $v$ of $\Sigma$ in $M$ and $g$ is the genus of $\Sigma$, then $\Sigma$ is a holomorphic curve.

Proof Let $K$ be the canonical line bundle over $\Sigma$, let $H^{0}(\Sigma, \mathcal{O}(v))$ denote the space of holomorphic sections of $v$ and let $H^{0}(\Sigma, \mathcal{O}(v \otimes K))$ denote the space of holomorphic sections of $v \otimes K$, then by Riemann-Roch theorem, we have

$$
\operatorname{dim} H^{0}(\Sigma, \mathcal{O}(v))=\chi(v)-g+1+\operatorname{dim} H^{0}(\Sigma, \mathcal{O}(v \otimes K)) \geq 1
$$

We therefore have a holomorphic section on $v$, so the corollary follows from Proposition 4.1.

\section{Symplectic Critical Surfaces in $\mathbf{C}^{2}$}

In this section, we construct some symplectic critical surfaces in $\mathbf{C}^{2}$ and prove a Liouville theorem for symplectic critical surfaces in $\mathbf{C}^{2}$. We start with a Liouville theorem. 
Theorem 5.1 If $\Sigma$ is a complete symplectic critical surface in $\mathbf{C}^{2}$ with area quadratic growth, and $\cos \alpha \geq \delta>0$, then it is a holomorphic curve.

Proof By the equation of the Kähler angle, we have ([3])

$$
\Delta \cos \alpha=\frac{3 \sin ^{2} \alpha-2}{\cos \alpha}|\nabla \alpha|^{2} .
$$

Thus,

$$
-\Delta \log \cos \alpha=2|\nabla \alpha|^{2} \text {. }
$$

We choose a cut-off function $\phi \in C_{0}^{\infty}\left(B_{2 R}(0)\right)$ such that $\phi \equiv 1$ in $B_{R}(0)$ and $|\nabla \phi| \leq$ $C / R$, where 0 is a point in $\Sigma$, and $B_{r}(0)$ is the metric ball centered at 0 with radius $r$. Multiplying $\phi^{2} \sin ^{2} \alpha$ on both sides of (5.2), integrating over $\Sigma$, one gets

$$
\begin{aligned}
2 \int_{\Sigma} \phi^{2}|\nabla \cos \alpha|^{2} d \mu & =\int_{\Sigma} \frac{\sin ^{2} \alpha}{\cos \alpha} \phi \nabla \phi \cdot \nabla \cos \alpha d \mu \\
& \leq C\left(\int_{\Sigma} \phi^{2}|\nabla \cos \alpha|^{2} d \mu\right)^{1 / 2}\left(\int_{B_{2 R}}|\nabla \phi|^{2} d \mu\right)^{1 / 2},
\end{aligned}
$$

which yields that

$$
\int_{\Sigma} \phi^{2}|\nabla \cos \alpha|^{2} d \mu \leq C,
$$

where we have used the assumption of the area quadratic growth. Letting $R \rightarrow \infty$, one gets that

$$
\int_{\Sigma}|\nabla \cos \alpha|^{2} d \mu \leq C
$$

We can rewrite the equation as

$$
\Delta \cos \alpha=\frac{3|\nabla \cos \alpha|^{2}}{\cos \alpha}-\frac{2|\nabla \cos \alpha|^{2}}{\sin ^{2} \alpha \cos \alpha} .
$$

Multiplying $\phi^{2}\left(1-\cos ^{2} \alpha\right)^{p} \cos \alpha$ on both sides of (5.4), where $p$ is a positive constant which will be decided later, integrating over $\Sigma$, we obtain

$$
\begin{aligned}
& \int_{\Sigma} \phi^{2}\left(1-\cos ^{2} \alpha\right)^{p}|\nabla \cos \alpha|^{2} d \mu-2 p \int_{\Sigma} \phi^{2} \cos ^{2} \alpha\left(1-\cos ^{2} \alpha\right)^{p-1}|\nabla \cos \alpha|^{2} d \mu \\
& =2 \int_{\Sigma} \phi^{2}\left(1-\cos ^{2} \alpha\right)^{p-1}|\nabla \cos \alpha|^{2} d \mu-3 \int_{\Sigma} \phi^{2}\left(1-\cos ^{2} \alpha\right)^{p}|\nabla \cos \alpha|^{2} \phi^{2} d \mu \\
& \quad-2 \int_{\Sigma} \cos \alpha \phi\left(1-\cos ^{2} \alpha\right)^{p} \nabla \cos \alpha \cdot \nabla \phi d \mu,
\end{aligned}
$$


and thus we have

$$
\begin{aligned}
& \int_{\Sigma} \phi^{2}\left[(2 p+4) \cos ^{2} \alpha-2\right]\left(1-\cos ^{2} \alpha\right)^{p-1}|\nabla \cos \alpha|^{2} d \mu \\
& \quad=2 \int_{\Sigma} \cos \alpha \phi\left(1-\cos ^{2} \alpha\right)^{p} \nabla \cos \alpha \cdot \nabla \phi d \mu \\
& \leq C \int_{B_{2 R} \backslash B_{R}}|\nabla \cos \alpha|^{2} d \mu .
\end{aligned}
$$

Applying (5.3) and letting $R \rightarrow \infty$, we know that the term on the right-hand side of the last inequality tends to zero. We choose $p$ such that $(2 p+4) \cos ^{2} \alpha-2 \geq$ $(2 p+4) \delta^{2}-2 \geq 1$, then we get that

$$
\int_{\Sigma}\left(1-\cos ^{2} \alpha\right)^{p-1}|\nabla \cos \alpha|^{2} d \mu=0,
$$

and it follows that $\nabla \cos \alpha \equiv 0$ on $\Sigma$ or $\cos \alpha=1$. This proves the theorem.

Then, we construct some symplectic critical surfaces in $\mathbf{C}^{2}$. We first consider the surface $\Sigma$ which is defined by

$$
F(u, v)=(v \cos u, v \sin u,-\ln v, 0), \quad u \in[0,2 \pi], \quad v>0
$$

It is in fact a rotational symmetric surface $z=-\frac{1}{2} \log \left(x^{2}+y^{2}\right)$ in $\mathbf{R}^{3}$, we consider it as a surface in $\mathbf{C}^{2}$.

It is clear that

$$
\begin{aligned}
& F_{v}=\left(\cos u, \sin u,-\frac{1}{v}, 0\right), \\
& F_{u}=(-v \sin u, v \cos u, 0,0) .
\end{aligned}
$$

We choose the normal basis

$$
\begin{aligned}
& v_{1}=\frac{1}{\sqrt{1+v^{2}}}(\cos u, \sin u, v, 0), \\
& v_{2}=(0,0,0,1) .
\end{aligned}
$$

Thus the induced metric on $\Sigma$ is

$$
\left(g_{i j}\right)=\left(\begin{array}{cc}
\frac{1+v^{2}}{v^{2}} & 0 \\
0 & v^{2}
\end{array}\right), \quad d \mu=\sqrt{1+v^{2}} d u d v
$$


It is evident that

$$
\begin{aligned}
& F_{v v}=\left(0,0, \frac{1}{v^{2}}, 0\right), \\
& F_{v u}=(-\sin u, \cos u, 0,0), \\
& F_{u u}=(-v \cos u,-v \sin u, 0,0) .
\end{aligned}
$$

Using the standard complex structure $J$ of $\mathbf{C}^{2}$,

$$
J=\left(\begin{array}{rrrr}
0 & -1 & 0 & 0 \\
1 & 0 & 0 & 0 \\
0 & 0 & 0 & -1 \\
0 & 0 & 1 & 0
\end{array}\right)
$$

the Kähler angle of $\Sigma$ is

$$
\cos \alpha=\frac{<J F_{v}, F_{u}>}{\sqrt{(\operatorname{det}(g))}}=\frac{v}{\sqrt{1+v^{2}}} .
$$

It is easy to compute that

$$
\begin{aligned}
H^{1} & =g^{11}<F_{v v}, v_{1}>+g^{22}<F_{u u}, v_{1}> \\
& =\frac{v}{\left(1+v^{2}\right)^{\frac{3}{2}}}-\frac{1}{v\left(1+v^{2}\right)^{\frac{1}{2}}} \\
& =-\frac{1}{v\left(1+v^{2}\right)^{\frac{3}{2}}}, \\
H^{2} & =g^{11}<F_{v v}, v_{2}>+g^{22}<F_{u u}, v_{2}> \\
& =0 .
\end{aligned}
$$

Since

$$
\begin{aligned}
\nabla \cos \alpha & =g^{11} \frac{\partial \cos \alpha}{\partial v} F_{v} \\
& =\frac{v^{2}}{1+v^{2}}\left(\frac{1}{\sqrt{1+v^{2}}}-\frac{v^{2}}{\left(1+v^{2}\right)^{\frac{3}{2}}}\right) F_{v} \\
& =\frac{v^{2}}{\left(1+v^{2}\right)^{\frac{5}{2}}} F_{v},
\end{aligned}
$$

we obtain that

$$
\begin{aligned}
(J \nabla \cos \alpha)^{\top} & =\frac{v^{2}}{\left(1+v^{2}\right)^{\frac{5}{2}}} \frac{<J F_{v}, F_{u}>F_{u}}{\left|F_{u}\right|^{2}} \\
& =\frac{v^{2}}{\left(1+v^{2}\right)^{\frac{5}{2}}} \frac{\cos \alpha \sqrt{\operatorname{det}(g)}}{v^{2}} F_{u}
\end{aligned}
$$




$$
\begin{aligned}
& =\frac{\cos \alpha}{\left(1+v^{2}\right)^{2}} F_{u}, \\
\left(J(J \nabla \cos \alpha)^{\top}\right)^{\perp} & =\frac{\cos \alpha}{\left(1+v^{2}\right)^{2}}\left[<J F_{u}, v_{1}>v_{1}+<J F_{u}, v_{2}>v_{2}\right] \\
& =-\cos \alpha \frac{v}{\left(1+v^{2}\right)^{\frac{5}{2}}} v_{1} .
\end{aligned}
$$

Therefore,

$$
\begin{aligned}
\cos ^{3} \alpha \vec{H} & =-\cos ^{3} \alpha \frac{1}{v\left(1+v^{2}\right)^{\frac{3}{2}}} v_{1} \\
& =-\cos \alpha \frac{v}{\left(1+v^{2}\right)^{\frac{5}{2}}} v_{1} \\
& =\left(J(J \nabla \cos \alpha)^{\top}\right)^{\perp} .
\end{aligned}
$$

So $\Sigma$ is a symplectic critical surface which is not minimal.

The Gauss curvature of $\Sigma$ is

$$
K=\frac{-1}{\left(1+v^{2}\right)^{2}} .
$$

It is not difficult to see that $\Sigma$ is a complete surface. See the Fig. 1.

We then consider the surface $\Sigma$

$$
F(u, v)=(v \sin u, v \cos u, f(v), u), \quad u \in(-\infty,+\infty), \quad v>0 .
$$

It is clear that

$$
\begin{aligned}
& F_{v}=\left(\sin u, \cos u, f_{v}, 0\right), \\
& F_{u}=(v \cos u,-v \sin u, 0,1)
\end{aligned}
$$

Fig. 1 Symplectic critical graph

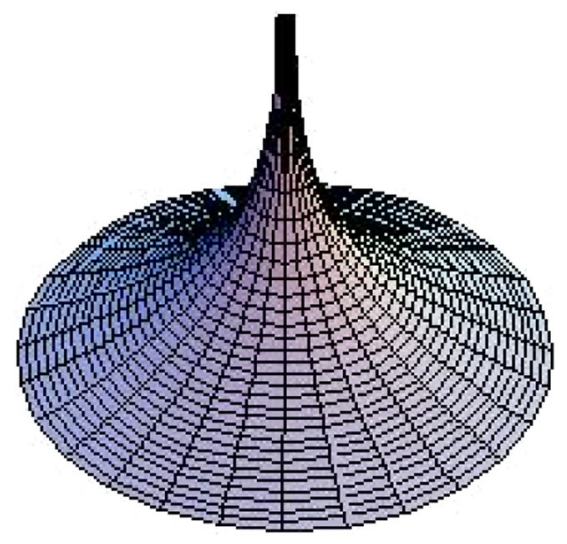


and

$$
d \mu=\sqrt{\left(1+v^{2}\right)\left(1+\left(f^{\prime}\right)^{2}\right)} d u d v .
$$

We choose the normal vectors

$$
\begin{aligned}
& v_{1}=\frac{1}{\sqrt{1+v^{2}}}(-\cos u, \sin u, 0, v), \\
& v_{2}=\frac{1}{\sqrt{1+\left(f^{\prime}\right)^{2}}}\left(f^{\prime} \sin u, f^{\prime} \cos u,-1,0\right) .
\end{aligned}
$$

Using the complex structure of (5.5), we have

$$
\cos \alpha=\frac{f^{\prime}-v}{\sqrt{\left(1+v^{2}\right)\left(1+\left(f^{\prime}\right)^{2}\right)}} .
$$

Straight computation yields that

$$
\begin{aligned}
& H^{1}=0, \\
& H^{2}=-\frac{f^{\prime \prime}}{\left(1+\left(f^{\prime}\right)^{2}\right)^{\frac{3}{2}}}-\frac{v f^{\prime}}{\left(1+v^{2}\right)\left(1+\left(f^{\prime}\right)^{2}\right)^{\frac{1}{2}}}
\end{aligned}
$$

and

$$
\left(J(J \nabla \cos \alpha)^{\top}\right)^{\perp}=\frac{\cos \alpha\left(1+v f^{\prime}\right)}{\left(1+\left(f^{\prime}\right)^{2}\right)\left(1+v^{2}\right)^{\frac{1}{2}}} \frac{\partial \cos \alpha}{\partial v} .
$$

One checks that $F$ is a symplectic critical surface in $\mathbf{C}^{2}$ if and only if $f(v)$ satisfies the equation

$$
\left(1+v^{2}\right)^{2} f^{\prime \prime}=1+\left(2 v-v^{3}\right) f^{\prime}+3 v^{2}\left(f^{\prime}\right)^{2}-v\left(f^{\prime}\right)^{3}
$$

It is clear that $f_{1}(v)=\frac{1}{2} v^{2}, f_{2}(v)=-\ln v$ and $f_{3}(v)=v^{2}+\ln v(v>0)$ are solutions. The surface $\Sigma$ with $f=f_{1}$ is Lagrangian which is not minimal. The surface $\Sigma$ with $f=f_{2}$ is a holomorphic curve. If we choose $f=f_{3}$, then $\Sigma$ is a symplectic critical surface which is not holomorphic.

Acknowledgments The research was supported the National Natural Science Foundation of China, No.11131007, No.11471014. The research was also supported by the Doctoral Programme Foundation of Institution of Higher Education of China, No. 20110002110064.

\section{References}

1. Chen, J., Tian, G.: Minimal surfaces in Riemannian 4-manifolds. Geom. Funct. Anal. 7, 873-916 (1997)

2. Chen, S.S., Wolfson, J.: Minimal surfaces by moving frames. Am. J. Math. 105, 59-83 (1983)

3. Han, X., Li, J.: Symplectic critical surface. J. Eur. Math. Soc. 12(2), 505-527 (2010)

4. Micallef, J., Wolfson, J.: The second variation of area of minimal surfaces in four-manifolds. Math. Ann. 295, 245-267 (1993) 